\title{
Therapien im Off-label-Use
}

\section{J. Dissemond}

\section{Off-label-Use zu Lasten der gesetzlichen Krankenkasse verordnungsfähig, wenn}

- Behandlung einer schwerwiegenden, lebensbedrohlichen oder die Lebensqualität auf Dauer nachhaltig beeinträchtigenden Erkrankung, und

- keine andere zugelassene Therapie verfügbar ist und

- aufgrund der Datenlage die begründete Aussicht besteht, dass mit dem Präparat ein Behandlungserfolg (kurativ oder palliativ) erzielt werden kann.

\section{Early Access:}

Früherer Zugriff auf noch nicht zugelassene Medikamente

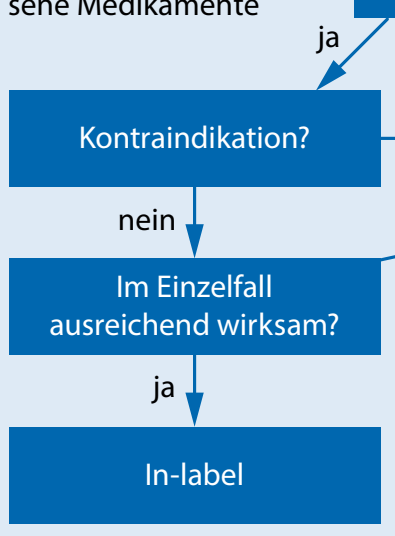

Für die Erkrankung zugelassene Therapie?

Compassionate Use: Einsatz nicht zugelassener Medikamente bei Patienten, die z.B. an lebensbedrohlicher Erkrankung leiden und mit zugelassenem o. genehmigten Arzneimittel nicht zufriedenstellend behandelt werden können

Phase-III-Studie oder veröffentlichte Erkenntnisse der

Wirksamkeit außerhalb eines Zulassungsverfahrens?

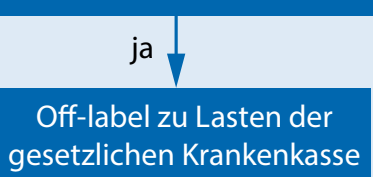
gesetzlichen Krankenkasse

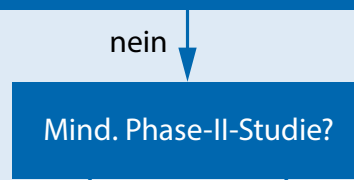

Lebensbedrohliche oder Lebensqualität nachhaltig beeinträchtigende Erkrankung? nein
Off-label nicht zu Lasten der gesetzlichen Krankenkasse

ja

Early Access

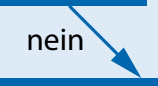

Compassionate Use

\section{Tipps für den Alltag}

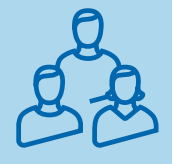

- Für viele dermatologische Krankheiten ist unter der aktuellen Off-label-Use-Regelung eine Verordnung zu Lasten der gesetzlichen Krankenkasse nicht möglich.

- Je nach Medikamentenpreis sollten Ärzte bei Off-label-Use einen Antrag auf Kostenübernahme bei der gesetzlichen Krankenkasse stellen, diese kann dann entscheiden.

- Ärzten und Patienten sollte bewusst sein, wenn Medikamente off-label eingesetzt werden

- Off-label-Use bei Therapieempfehlungen in Leitlinien und Lehrbüchern eindeutig kennzeichnen.

- Ärzte sollten regelmäßig über aktuelle Entwicklungen zu Compassionate Use und Early-access-Programmen informiert werden.

\section{Springer Medizin}

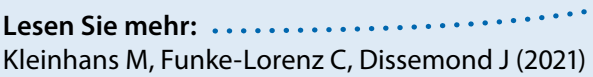

www.springermedizin.de/link/19090630 